\title{
Macular Telangiectasia: A cause of bull's eye maculopathy
}

\author{
Sara Fernandez Schultis and Chan Nguyen* \\ The University of Texas Southwestern Medical Center, Dallas, USA
}

\begin{abstract}
A 52-year-old woman with blurred vision was found to have a bull's eye lesion in both eyes. On optical coherence tomography, she was found to have outer retinal atrophy with inner subfoveal cysts. A reduction in the normal foveal hypoautofluorescence and abnormalities on multifocal electroretinography were also observed. Visual field testing, fluorescein angiography, electrooculography, and full-field electoretinography were also conducted. Her findings are consistent with a diagnosis of Macular Telangiectasia type 2 and this case demonstrates the importance of keeping Macular Telangiectasia type 2 on the differential for patients demonstrating a bull's eye maculopathy.
\end{abstract}

\section{Introduction}

Macular Telangiectasia Type 2 (MacTel) is a bilateral disease with abnormalities of the neurosensory retina and juxtafoveolar capillaries which presents with slow loss of central visual acuity [1]. Though the exact cause is still unknown, it is marked by atrophy of foveal photoreceptors and vascular leakage due to vessel abnormalities. Patients tend to present with visual complaints once they have reached the fifth or sixth decade of life [2]. Paracentral scotomas and metamorphopsia may be present in later stages of the disease. One population-based study of adults age 43-86 years suggests that MacTel affects about $0.1 \%$ of individuals [3]. MacTel is distinct from the less common Macular Telangiectasia Type 1 which presents with congenital unilateral abnormalities involving microaneurysms of retinal vessels.

MacTel initially presents with abnormalities in the temporal paracentral regions of the retina, but findings may be subtle. This can progress to form an oval or circular diseased region centered around the foveola with the horizontal raphe not respected. Fundoscopic findings include a lack of foveolar reflex, retinal graying, diving right-angle vessels, superficial crystalline deposits, and retinal pigment plaques. On optical coherence tomography (OCT), large cavitary cysts can form in the retina leaving behind only the inner limiting membrane (ILM), an appearance described as an ILM drape. In advanced cases, full thickness macular holes and neovascular complexes can form. Areas of retinal pigment epithelium hyperplasia appear as hypofluorescent spots on fundus autofluorescence (FAF). Overall foveal FAF, however, is pathognomonically increased in MacTel, meaning that is there is a loss of the physiologic foveal hypoautofluorescence. Nasal regions of the retina can be, but are often unaffected, and peripheral visual field defects are not a common complaint [2]. One study found baseline mean visual acuity in $522 \mathrm{MacTel}$ eyes was 20/40, with about half of the eyes having visual acuity of 20/32 or better [4]. Even in advanced disease, visual acuity below 20/200 is rare [2]. Diagnosis is made by combining clinical experience with imaging methods such as fluorescein angiography (FA), OCT, and FAF.

There is currently no successful treatment shown to improve visual acuity in patients with non-proliferative MacTel though focal argon laser photocoagulation, photodynamic therapy, intravitreal injection of triamcinolone, and anti-VEGF therapies have been studied [3]. In the proliferative stage of MacTel marked by subretinal neovascularization, intravitreal anti-VEGF therapy such as bevacizumab can potentially improve visual acuity [3].

Our report will describe a presentation of MacTel as a bull's eye maculopathy with FA, OCT, FAF, visual field testing, and electrophysiologic studies. The differential diagnosis of bull's eye maculopathy commonly includes drug induced- hydroxychloroquine or chloroquine retinopathy, Stargardt disease, cone dystrophy, and benign concentric macular dystrophy. MacTel should be added to the differential for bull's eye maculopathy.

\section{Case report}

A 52-year-old Hispanic female presented to the Parkland Hospital Eye Clinic with complaints of blurred vision predominantly in her right eye. She had a past ophthalmologic history of bilateral incipient cataracts. She was referred for a diabetic eye exam as she had been recently diagnosed with type 2 diabetes mellitus three months prior. The patient noted that blurred vision had begun two months prior to her eye clinic visit but that it had been gradually improving. She reported difficulties with near vision, but not with distance vision. She denied scotoma, nyctalopia and had no history of chloroquine or hydroxychloroquine ingestion. She reported no other family members with visual difficulties.

The patient's best corrected visual acuity was 20/50 in the right eye and 20/25 in the left eye. Anterior segment exam and intraocular pressure values were within normal limits in both eyes. On retinal exam both eyes demonstrated a concentric defect in the macula with depigmentation of the retinal pigment epithelium, parafoveal

Correspondence to: Chan Nguyen, 5323 Harry Hines Blvd, Dallas, TX 753909057, USA, Tel: 214-648-4733; Fax: 214-648-2270; E-mail: Chan.Nguyen@ utsouthwestern.edu

Key words: bull's eye maculopathy, electrophysiological tests, fluorescein angiography, fundus auto fluorescence, Macular Telangiectasia Type 2, optical coherence tomography (OCT)

Received: September 28, 2017; Accepted: October 13, 2017; Published: October 16, 2017 
graying, and loss of retinal transparency (Figure 1). Within the ring of depigmentation, the right eye had a few spots of pigment clumping. The peripheral retina was normal.

Fluorescein angiography showed parafoveal annular staining with central sparing, right eye worse than the left (Figure 2). In both eyes, fine retinal telangiectatic vessels were noted parafoveally and leakage was particularly prominent temporal to the fovea. The fluorescein staining pattern in the right eye was mildly blocked by the pigmented deposits.

On OCT, the right eye showed diffuse central outer retinal atrophy with collapse of the inner retina posteriorly, resulting in a cystic appearance under the inner limiting membrane (Figure 3). The left eye demonstrated similar but less severe findings, manifesting as patchy outer retinal atrophy with smaller inner retinal cysts. OCT through the pigmented spot on the right eye revealed hyperreflective material in the middle retina. When divided into the nine Early Treatment Diabetic Retinopathy Study subfields, the Spectralis-measured central subfields showed retinal thinning bilaterally (right, $246 \mu \mathrm{m}$; left, $231 \mu \mathrm{m}$; normal $269 \pm 22 \mu \mathrm{m}$ ) [5]. The inner macular subfields showed more significant thinning on the right eye (271-284 $\mu \mathrm{m})$ than the left $(280-309 \mu \mathrm{m})$ (normal, 324-337 $\pm 13-23 \mu \mathrm{m}$ ) [5].

Fundus autofluorescence revealed discrete patches of focal parafoveal hypoautofluorescence in the right eye (Figure 4) corresponding to the hyperreflective deposits on OCT and the pigmented lesions on funduscopy. In addition, both eyes demonstrated a reduction in the normal foveal hypoautofluorescence.

Visual field testing in the right showed mild nonspecific paracentral superior defects with a mean deviation of -0.51 and fixation loss of $5 / 13$. Testing in the left eye was reliable and within normal limits.

The Arden ratio on electrooculogram was normal at $298 \%$ for the right eye and 355\% for the left eye. Full-field electroretinography (ERG) and bright flash oscillatory potentials were normal in both eyes. The ring ratio on multifocal ERG was abnormal in both eyes with a reduced foveal amplitude in the right eye and a normal foveal amplitude in the left eye (Figure 5).

Fundus examination of her three children ages 16, 22, and 27 and one 9-year-old grandchild revealed no abnormalities. All family members had normal best-corrected visual acuity and none had any vision complaints.

\section{Differential diagnosis}

The differential diagnosis for a bull's eye maculopathy commonly includes hydroxychloroquine or chloroquine toxicity, Stargardt

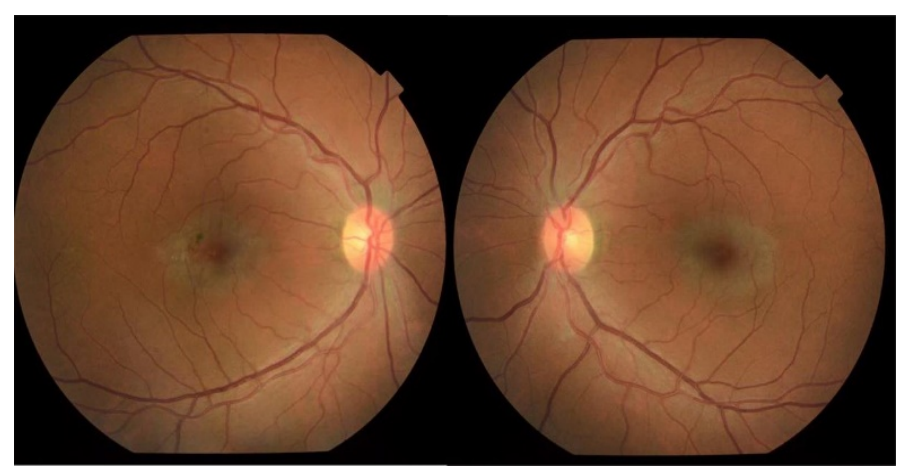

Figure 1. Funduscopy: right eye (left), left eye (right)

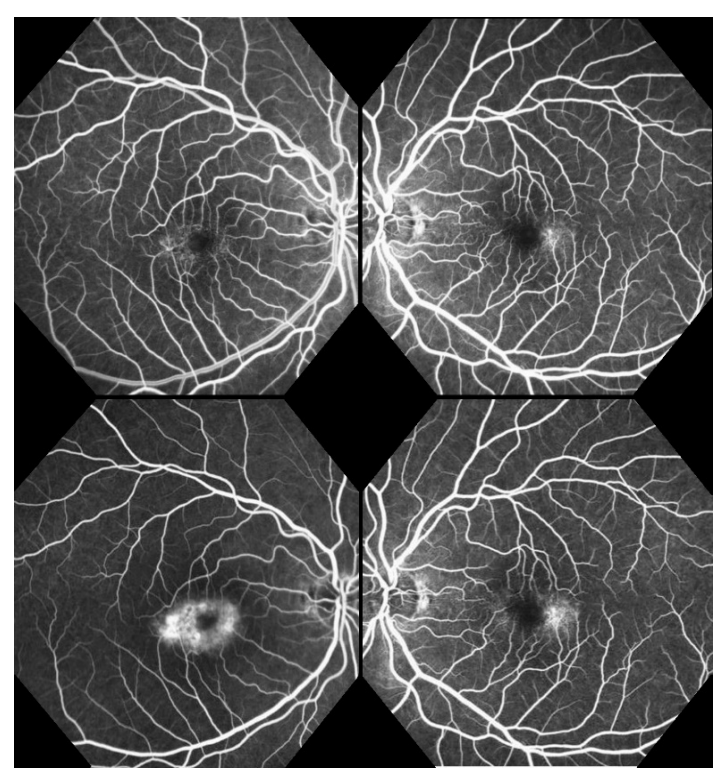

Figure 2. Fluorescein angiography: early phase (top), late phase (bottom)

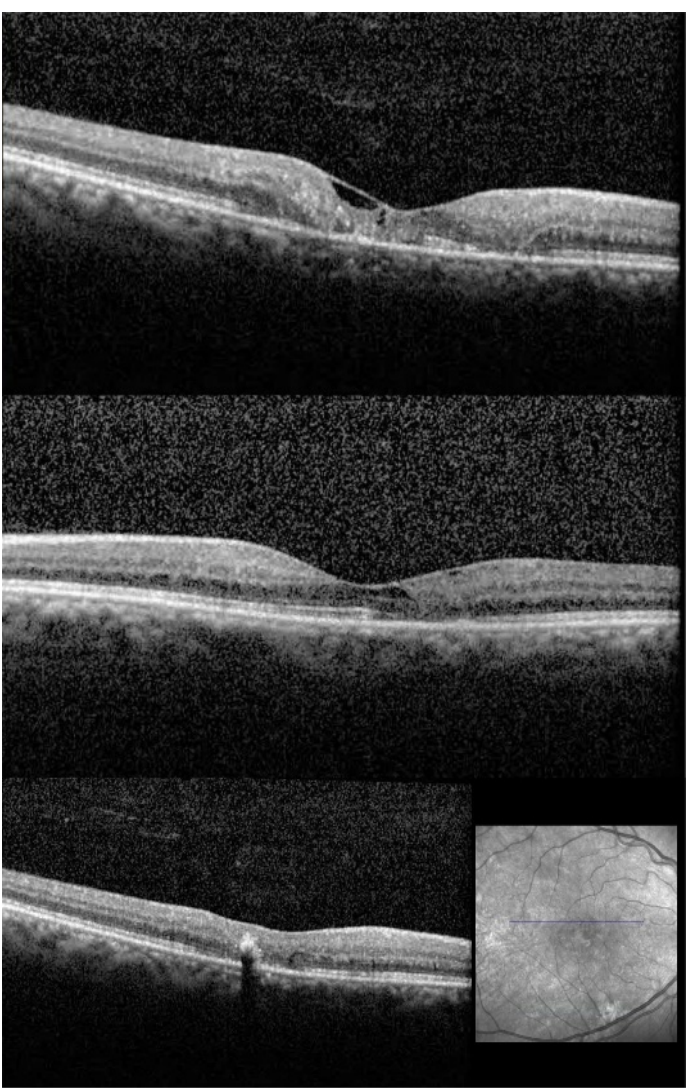

Figure 3. Optical coherence tomography: right eye (top), left eye (middle), right eye through pigmented lesion (bottom)

disease, cone dystrophy, cone-rod dystrophy, and benign concentric macular dystrophy.

Hydroxychloroquine or chloroquine ingestion can cause atrophy of the retinal pigment epithelium in the form of a bull's eye maculopathy in cases of chronic use. Early detection of toxicity prior to the development of the bull's eye is important, as loss of visual acuity is usually irreversible at that stage. 


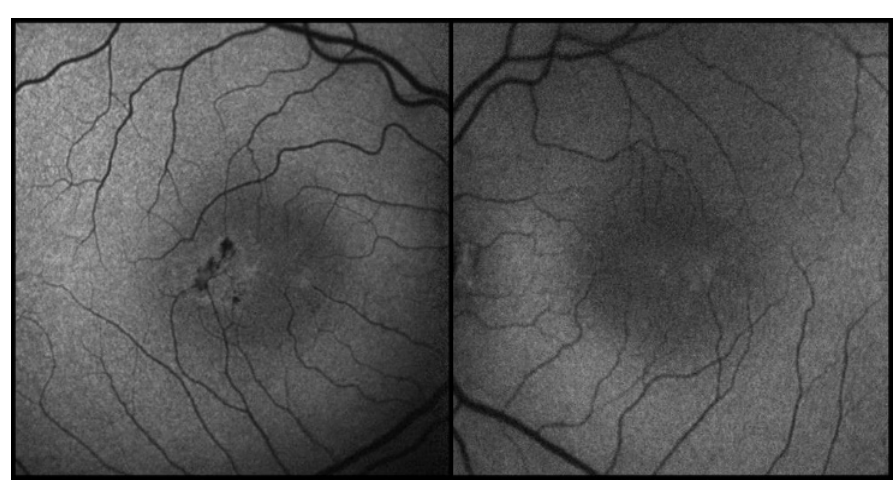

Figure 4. Fundus autofluorescence: right eye (left), left eye (right)

\begin{tabular}{|c|c|c|}
\hline \multicolumn{3}{|c|}{ Ring Ratios } \\
\hline \multirow{2}{*}{ Ring (n) } & \multicolumn{2}{|c|}{ R1/Rn P1 Amp } \\
\cline { 2 - 3 } & Right Eye & Left Eye \\
\hline 1 & 1.00 & 1.00 \\
\hline 2 & 1.41 & 1.94 \\
\hline 3 & 2.36 & 3.33 \\
\hline 4 & 3.03 & 4.47 \\
\hline 5 & 3.36 & 5.20 \\
\hline
\end{tabular}
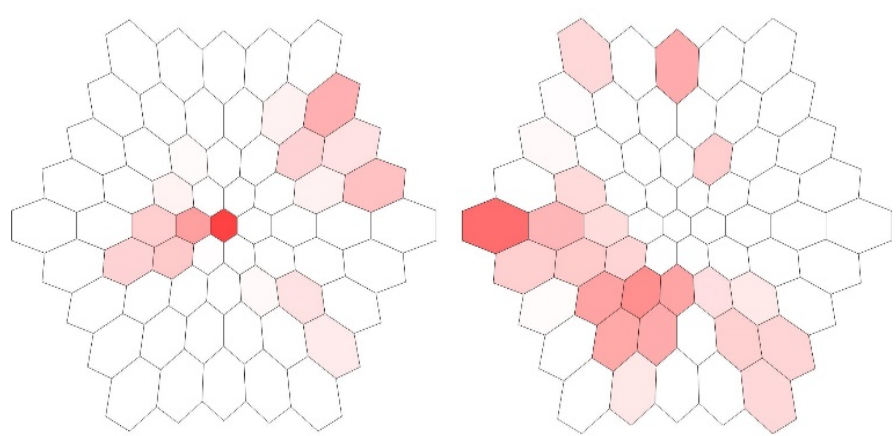

Right

Left

Eye

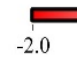

-1.0
Standard

Deviation

Figure 5. Multifocal electroretinography: ring ratios (top), 2D topography (bottom)

Stargardt disease is a relatively common hereditary macular dystrophy associated with loss of visual acuity typically starting in childhood or early adulthood [6]. On funduscopy, the characteristic finding is yellow to grey pisciform flecks in the posterior pole. Atrophy of the central macula and a silent choroid on fluorescein angiography are commonly seen [6]. Cone dystrophies manifest as decreased visual acuity with central scotomas, reduced colour vision, and photophobia. Bull's eye maculopathy can be seen on fundus exam often following the onset of vision changes [6]. ERG will show a decrease in cone-mediated responses with normal scotopic ERG findings [7]. As in Stargardt disease, patients with a progressive cone dystrophy tend to present in late childhood and young adulthood. Patients initially diagnosed with cone dystrophy may progress to cone-rod dystrophy. Rod dysfunction, detectable on scotopic ERG testing, leads to night blindness and loss of peripheral vision [6].

Benign concentric annular macular dystrophy (BCAMD) is a rare bull's eye maculopathy that involves relatively good visual acuity. It is an autosomal dominant disease and there are only a handful of published case reports and a series of reports following a three-generation family with BCAMD to date.
The listed diagnoses can be ruled out as our patient denied prior use of hydroxychloroquine or chloroquine, ruling out retinal toxicity from these medications. She did not demonstrate pisciform flecks with a silent choroid on fluorescein angiogram to suggest Stargardt disease. In addition, her electrophysiological studies were not consistent with a cone dystrophy or a cone-rod dystrophy, nor did she present with symptoms that are seen with cone dystrophies such as defective colour vision and photophobia. BCAMD is less likely due to the extreme rarity of the disease and the lack of family history. Her imaging findings including parafoveal graying, telangiectatic vessels, and FA leakage particularly prominent temporal to the fovea, together with the spots of RPE hyperplasia and ILM drape, make MacTel the likely diagnosis.

\section{Discussion}

Patients with MacTel can have variable clinical presentations, but maintenance of relatively good visual acuity in the presence of parafoveal graying, telangiectasias, and temporally located macular hyperfluorescence on angiography is characteristic. MacTel may progress from temporally located lesions to form an oval or circular diseased region parafoveally in a manner that appears as a bull's eye maculopathy. Early studies illustrated that paracentral angiographic leakage on FA involved greater than $270^{\circ}$ in $29 \%$ of $182 \mathrm{MacTel}$ eyes analysed [1]. In the present case, the patient's condition appears to affect her right eye more than her left; however, she has bilateral bull's eye maculopathy illustrated on funduscopic exam and fluorescein angiography. The observed hypoautofluorescent patches in the right eye correspond with hyperreflective deposits seen on OCT and pigmented areas seen on fundus exam. Small patches of abnormal retinal pigmentation can develop in response to photoreceptor degeneration from any number of causes. The generalized bilateral foveal hyperautofluorescence likely represents changes in composition to the retinal pigment epithelium [8].

It will be important to monitor the patient's children for symptoms as they reach their fifth or sixth decade of life, as this is when characteristic retinal findings associated with MacTel begin. Though there is no current causal gene candidate for MacTel, it is believed that genetic factors with variable expressivity underlie the disease. Studies of affected families suggest autosomal dominant inheritance with reduced penetrance [9]. According to the MacTel Project [10], it is estimated that MacTel may develop in between $10-50 \%$ of an affected person's first-degree relatives. Though true cause of MacTel is unknown, current research suggests a decreased number and/or dysfunction of Müller cells in the central macula plays a role in disease pathogenesis, as these cells have neuroprotective functions as well as an intimate relationship with retinal vessels [11].

\section{References}

1. Gass JD, Blodi BA (1993) Idiopathic juxtafoveolar retinal telangiectasis: update of classification and follow-up study. Ophthalmology 100: 1536-1546. [Crossref]

2. Charbel Issa P, Gillies MC, Chew EY, Bird AC, Heeren TF, et al. (2013) Macular telangiectasia type 2. Prog Retin Eye Res 34: 49-77. [Crossref]

3. Klein R, Blodi BA, Meuer SM, Myers CE, Chew EY, et al. (2010) The prevalence of macular telangiectasia type 2 in the Beaver Dam eye study. Am J Ophthalmol 150: 55-62. [Crossref]

4. Clemons TE, Gillies MC, Chew EY, Bird AC, Peto T, et al. (2010) Baseline characteristics of participants in the natural history study of macular telangiectasia (MacTel) MacTel Project Report No. 2. Ophthalmic Epidemiol 17: 66-73. [Crossref]

5. Grover S, Murthy R, Brar V, Chalam K (2009) Normative Data for Macular Thickness by High-Definition Spectral-Domain Optical Coherence Tomography (Spectralis). Am J Ophthalmol 148: 266-271. [Crossref] 
6. Holz F, Spaide R (2005) Medical Retina. 1st ed. Springer Berlin Heidelberg.

7. Krill AE, Deutman AF, Fishman M (1973) The cone degenerations. Doc Ophthalmol 35: 1-80. [Crossref]

8. Wong WT, Forooghian F, Majumdar Z, Bonner RF, Cunningham D, et al. (2009) Fundus autofluorescence in type 2 idiopathic macular telangiectasia: correlation with optical coherence tomography and microperimetry. Am J Ophthalmol 148: 573-583. [Crossref]
9. Nancy L. Parmalee, Carl Schubert, Maria Figueroa, Alan C. Bird, Tunde Peto, et al. (2012) Identification of a Potential Susceptibility Locus for Macular Telangiectasia Type 2. PLoS ONE 7: e24268 [Crossref]

10. About Macular Telangiectasia (MacTel). The Lowy Medical Research Institute. http:// www.lmri.net/mactel/macular-telangiectasia-mactel/ (accessed 21 Jul 2017).

11. Powner MB, Gillies MC, Tretiach M, Scott A, Guymer RH, et al. (2010) Perifoveal Müller Cell Depletion in a Case of Macular Telangiectasia Type 2. Ophthalmology 117 2407-2416. [Crossref]

Copyright: $\odot 2017$ Schultis SF. This is an open-access article distributed under the terms of the Creative Commons Attribution License, which permits unrestricted use, distribution, and reproduction in any medium, provided the original author and source are credited. 